\title{
Mechanisms of Lumen Development in Drosophila Tubular Organs
}

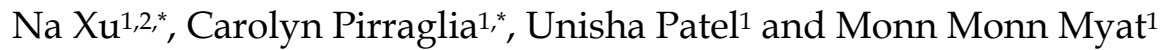 \\ ${ }^{1}$ Department of Cell and Developmental Biology, Weill Medical \\ College of Cornell University, New York \\ ${ }^{2}$ Department of Cell Biology, Albert Einstein College of Medicine, New York
}

USA

\section{Introduction}

Tubular organs of both invertebrate and vertebrate animals serve many important physiological functions, such as the delivery of gases, nutrients and hormones and removal of waste. All tubular organs contain a central lumen that is formed through a variety of mechanisms and whose size and shape is essential for organ function. While some lumens form from pre-polarized cells, others form de novo from single cells or solid cords of cells (Andrew and Ewald, 2010). Studies of lumen formation in tubular organs in the Drosophila embryo have benefited from the genetic analysis available in Drosophila and the advent of sophisticated microscopic techniques that allow lumen formation to be visualized in vivo in real time in a developing embryo. In this chapter we will review recent advances on the cellular and molecular mechanisms by which lumens form and their size is controlled in the salivary gland, trachea and dorsal vessel of the Drosophila melanogaster embryo.

\section{Dorsal vessel}

The Drosophila cardiac tube, or dorsal vessel, is a hemolymph pumping organ that constitutes the entire cardiovascular system of the Drosophila open circulatory system. The dorsal vessel is established during embryogenesis and is composed of two rows of 52 contractile myoendothelial cells (cardioblasts [CBs]) enclosing a central lumen surrounded by loosely attached non-muscular pericardial cells (Figure 1A and B) (Tao and Schulz, 2007). The dorsal vessel is derived from mesodermal cells that acquire certain epithelial characteristics to form two bilateral rows of CBs that migrate dorsally and meet at the dorsal midline to create a lumen exclusively formed by the membrane walls of the CBs (Figure 1B). At the end of cardiac morphogenesis, the posterior portion of the dorsal vessel becomes enlarged and constitutes the definitive heart, whereas the anterior portion has a narrow diameter and is equivalent to the aorta (Figure 1A). The heart is the only region of the dorsal vessel that exhibits automatic and synchronized beating to act as a myogenic pump and promote circulation of the hemolymph throughout the cardiovascular system.

* These authors contributed equally 
In this section, we discuss the genetic networks that control lumen formation of the Drosophila dorsal vessel. In particular, we discuss the necessary changes in cell shape and cell-cell adhesion that occur during lumen formation, and the requirement of G-protein signaling for maintenance of the cardiac tube.

\subsection{Dorsal vessel lumen formation}

The cardiac myoendothelium originates from mesodermal cells that form two bilateral rows of CBs. During dorsal closure, when the dorsal epidermis from opposing sides of the embryo migrates as a sheet to seal the opening at the dorsal surface, the two rows of aligned CBs, together with adjacent pericardial cells, migrate as a sheet of cells, in association and in coordination, with the overlying ectoderm towards the dorsal midline. Lateral alignment and dorsal migration of CBs are critical for the proper formation of the mature dorsal vessel, as mutations in genes that regulate these processes result in structural and lumenal defects (Reim and Frasch, 2010; Tao and Schulz, 2007). As the lateral rows of CBs approach the dorsal midline, the CBs adopt a pear-like shape through constriction of their cellular surfaces facing the dorsal midline (Figure 1C) (Medioni et al., 2008; Santiago-Martinez et al., 2008). Actin-rich protrusions extend from this membrane domain, which constitutes the leading edge of the dorsally migrating CBs (Medioni et al., 2008). CBs from each of the two lateral rows initiates contact with its contralateral counterpart at their dorsal-most leading edge and join at the dorsal midline. Subsequently, the CBs adopt a crescent-like shape, thereby allowing contralateral CBs to join ventrally to close the tube and form a central lumen (Figure 1C) (Medioni et al., 2008; Santiago-Martinez et al., 2008).
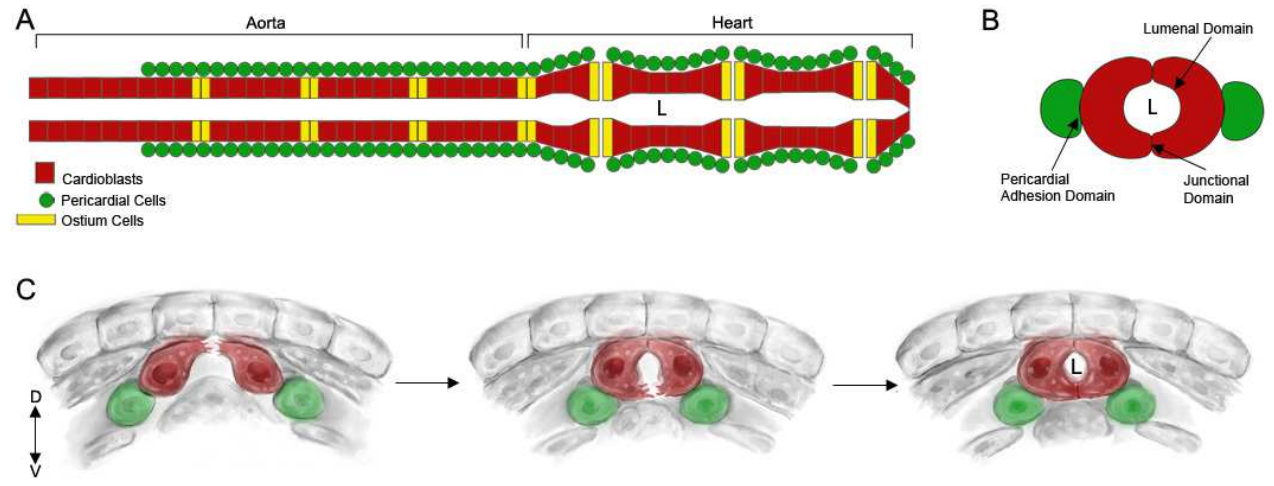

Fig. 1. Lumen formation in the dorsal vessel.

(A) The Drosophila embryonic dorsal vessel consists of the aorta and the heart proper where the lumen is lined by cardioblasts (red) and ostium cells (yellow) which in turn are surrounded by the pericardial cells (green). (B) Cross section of the dorsal vessel with a central lumen (L) showing the lumenal and junctional domains of the cardioblasts (red) and the pericardial adhesion domain between the cardioblasts and pericardial cells (green). (C) Lumen formation in the dorsal vessel is preceded by the dorsal migration of a row of cardioblasts (red) in contact with pericardial cells (green) on each side of the embryo, followed by cell-cell contact at the dorsal and then the ventral sides of the cardioblasts to form a central lumen. D: dorsal; V: ventral. Panel C was kindly provided by F. Macabenta and S. Kramer. 
Concomitant with these cell shape changes, the membrane domains of CBs undergo significant remodeling to alter their cellular polarity. As CBs approach the dorsal midline, proteins required for cell adhesion, junctional domain formation and attractive/repulsive signals become sub-localized within distinct CB membrane domains. The lumenal domain, which encloses the lumen itself, is characterized by the presence of basal membrane matrix proteins, including Dystroglycan and Perlecan, and the attractant/repellant proteins, Slit and Roundabout (Robo) (Figure 1B). The junctional domain, located at the ventral and dorsal membrane regions, is characterized by the accumulation of adherens junction (AJ) proteins, such as E-Cadherin, Discs-Large and $\beta$-catenin. The pericardial adhesion domain, which exists at the contact points between the CBs and the pericardial cells (PCs), is distinguished by the presence of extracellular matrix (ECM) proteins, such as pericardin (Chartier et al., 2002).

The specification and maintenance of these distinct membrane domains and the dynamic changes in cell shape require specific genetic regulators, the loss of which disrupts lumen formation. Shotgun (shg), which encodes the Drosophila homolog of E-cadherin, is specifically required for adhesion of opposing rows of CBs to form the junctional domain (Haag et al., 1999; Santiago-Martinez et al., 2008). The loss of shg function results in a loss of adhesion between contralateral CBs, whereas the overexpression of E-cadherin in CBs results in an expansion of the junctional domain and the inability to form a lumen (Haag et al., 1999; Santiago-Martinez et al., 2008). One key regulator of E-cadherin-mediated adhesion between contralateral CBs is the Slit/Robo signaling pathway. Slit is an EGF- and LRR-containing secreted extracellular matrix protein that functions as the ligand for the Robo family of transmembrane receptors and has been shown previously to regulate repulsive axonal guidance in the Drosophila nervous system (Kidd et al., 1999; Qian et al., 2005; Rothberg et al., 1990). During migration of the bilateral rows of CBs, Slit and Robo accumulate at the presumptive lumenal domain as the CBs align at the dorsal midline. This polarization of Slit/Robo signaling is critical for its function in regulating lumen formation. In robo and slit mutants, E-cadherin mediated adhesion between the two opposing CBs is expanded preventing critical cell shape changes and blocking lumen formation (Medioni et al., 2008; Santiago-Martinez et al., 2008). In contrast, when Slit is ectopically expressed on all CB surfaces, a loss of cell adhesion was observed, resulting in the formation of multiple lumens (Santiago-Martinez et al., 2008). These studies indicate that polarized Slit/Robo repulsion is required for inhibition of E-cadherin mediated adhesion at the presumptive lumenal domain to form a central lumen.

Restriction of Slit localization to the lumenal domain is regulated by the transmembrane heparin sulfate proteoglycan, Syndecan (Knox et al., 2011). Syndecans are known to interact with a diversity of extracellular ligands, often in conjunction with other cell surface receptors, and are thought to play a dual role in adhesion and as regulators of signaling from the extracellular matrix (ECM). In Drosophila, the single Syndecan homolog, Sdc, regulates axon guidance by acting as a co-receptor with Robo to mediate Slit signaling (Chanana et al., 2009). Embryos that lack Sdc function fail to localize Slit and Robo to the luminal domain and fail to properly form a lumen, indicating that Sdc may also act as a coreceptor for Slit to regulate lumen formation in the dorsal vessel (Knox et al., 2011).

Formation of the dorsal vessel lumen also depends on the transmembrane receptor, Uncoordinated 5 (Unc5). Unc5 represents the single Drosophila homolog of a conserved 
receptor family that binds to the secreted ligand, Netrin (Net) (Keleman and Dickson, 2001). Unc5/Net signaling, like Slit/Robo signaling, plays a role in repulsive axonal guidance and has a localization pattern in the dorsal vessel similar to that of Slit and Robo, where Unc5, and its ligand, NetB, accumulate at the lumenal domain of CBs (Albrecht et al., 2011; Keleman and Dickson, 2001; von Hilchen et al., 2010). In embryos mutant for unc5 or netB, CBs migrate and initiate contact with their contralateral counterparts normally but fail to form a central lumen (Albrecht et al., 2011). Thus, Unc5/Netrin acts as a repulsive force to inhibit contralateral CBs from attaching to one another at their presumptive lumenal domains.

\subsection{Maintenance of the dorsal vessel}

Genetic analysis has identified the mechanisms by which the lumen of the dorsal vessel is maintained. In particular, at the end of dorsal vessel development, the pericardial cells and CBs must adhere tightly to maintain the structure and integrity of the dorsal vessel. The loss of pericardial and CB adhesion results in the disruption of the dorsal vessel lumen and loss of cardiac function (Yi et al., 2006). The mevalonate pathway, which is important for the synthesis of isoprene derivatives that modify the $C$ termini of proteins containing a CAAX motif ( $C$, cysteine; A, aliphatic amino acids; $X$, any amino acid), is required for proper pericardial and $\mathrm{CB}$ adhesion and dorsal vessel maintenance. In mutants for HMGCR (hydroxymethylglutaryl (HMG)-coenyme A (CoA) reductase), an important regulator of the mevalonate pathway, CBs and pericardial cells properly align at the dorsal midline to form a central lumen; however, at the end of embryogenesis, pericardial cells dissociate from the CBs resulting in CB misalignment and loss of lumen integrity (Yi et al., 2006).

Dorsal vessel defects of HMGCR mutant embryos result from the failure of $\mathrm{G}$ protein $\gamma$ subunit $1(\mathrm{G} \gamma 1)$ to be post-translationally modified with a geranylgeranyl moiety (Yi et al., 2006; Yi et al., 2008). G proteins form heterotrimers with subunits designated $\alpha, \beta$ and $\gamma$ and act as intracellular effectors of $G$ protein coupled receptors (GPCRs) (Malbon, 2005). G $\gamma 1$ functions with the $\beta$ and a subunits, G $\beta 13 \mathrm{~F}$ and G-oa47A, respectively, to regulate dorsal vessel maintenance, where loss of G $\beta 13 \mathrm{~F}$ or G-oa47A results in pericardial cell-CB dissociation (Yi et al., 2008). Genetic analysis indicates that regulation between the Ga and $\mathrm{G} \beta Y$ subunits, in coordination with Loco, a member of the regulators of G-protein signaling (RGS) protein family, ensure proper maintenance of the dorsal vessel (Yi et al., 2008). One mechanism by which heterotrimeric G proteins regulate CB-pericardial cell adhesion is by regulating septate junction (SJ) components (Yi et al., 2008). In Drosophila, SJs are spoke and ladder septa that connect adjacent plasma membranes and are functionally similar to tight junctions in mammalian systems (Banerjee et al., 2006). Although SJs are absent in the embryonic dorsal vessel, SJ proteins are present, suggesting that SJ proteins perform non-canonical functions during dorsal vessel morphogenesis. Gy1 regulates the cellular localization of the SJ proteins, Coracle (Cora), Sinuous (Sinu), Neurexin-IV (Nrx-IV) and Nervana2 (Nrv2), and mutants for these SJ proteins have defects in pericardial cell-CB adhesion (Yi et al., 2008). In embryos mutant for SJ proteins, CBs properly align and adhere their ventral and lateral membrane domains at the dorsal midline to form a central lumen; however, the lumen is not maintained and becomes twisted and flattened ( $\mathrm{Yi}$ et al., 2008). This is in contrast to embryos mutant for AJ proteins, where $\mathrm{CBs}$ fail to initialize adhesion with contralateral CBs at the dorsal midline. 
These studies suggest a novel pathway in which heterotrimeric G-protein signaling controls proper localization and function of SJ proteins at the pericardial adhesion domain of CBs, which leads to the establishment of stable "SJ-like" adhesive contacts with pericardial cells to maintain the mature dorsal vessel lumen.

\section{Salivary gland}

The Drosophila salivary gland is a secretory organ and consists of a pair of elongated secretory tubes (hereafter referred to the salivary gland) that are connected to the larval mouth through the finer set of duct tubes. The glands are formed during embryogenesis and become functional in the larval stage when they synthesize and secrete proteins necessary for lubrication, digestion and taste. The salivary gland consists of a layer of polarized epithelial cells surrounding a central lumen that is formed from two placodes of epithelial cells, approximately 100 cells each. Salivary glands invaginate through constriction of apical domains and basal migration of nuclei to form a tube that is initially oriented dorsally. After all salivary gland cells have invaginated, the gland turns and migrates posteriorly until it reaches its final position in the embryo (Pirraglia and Myat, 2010). In this section, we will focus on our current understanding of how the salivary gland lumen achieves and maintains its size and shape.

\subsection{Growth and remodeling of the apical membrane}

The salivary gland lumen forms concomitantly with invagination of gland cells from the embryo surface. During the early migratory step of salivary gland development when the internalized gland turns and migrates posteriorly, gland lumen length doubles and lumen width in the proximal region (the region closest to the ventral surface) is reduced by half (Figure 2A-C) (Pirraglia et al., 2010). Salivary gland lumen size is controlled, at least in part, by the dynamic growth and remodeling of the apical membrane. Transmission electron micrographs (TEMs) revealed that after all salivary gland cells have invaginated from the embryo surface, the gland lumen is characterized by abundant apical protrusions into the luminal space (Myat and Andrew, 2002). Measurements of the length of the apical surface membrane per individual salivary gland cell showed an increase in apical surface membrane, suggesting dramatic growth of the apical membrane (Myat and Andrew, 2002). This rapid phase of membrane growth is followed by elongation of the apical domain of individual gland cells in the proximal-distal (Pr-Di) direction, the direction in which the salivary gland lumen elongates concomitant with posterior migration of the gland (Figure 2D) (Myat and Andrew, 2002; Pirraglia et al., 2010). The Sp1/egr-like transcription factor, Huckebein $(\mathrm{Hkb})$, regulates the size and shape of the salivary gland lumen through control of apical membrane growth (Myat and Andrew, 2000a; Myat and Andrew, 2002). In $h k b$ mutant salivary gland cells, the apical surface membrane fails to grow and the apical domain fails to elongate resulting in spherical lumens (Myat and Andrew, 2002).

In the salivary gland placode, the pattern of $h k b$ RNA precedes the order in which salivary gland cells invaginate (Myat and Andrew, 2000a). This pattern of $h k b$ RNA expression is controlled by Hairy, a basic helix-loop-helix (bHLH) transcription factor (Carroll et al., 1988; Hooper et al., 1989). In hairy mutant embryos, $h k b$ RNA is expressed in all gland cells and 

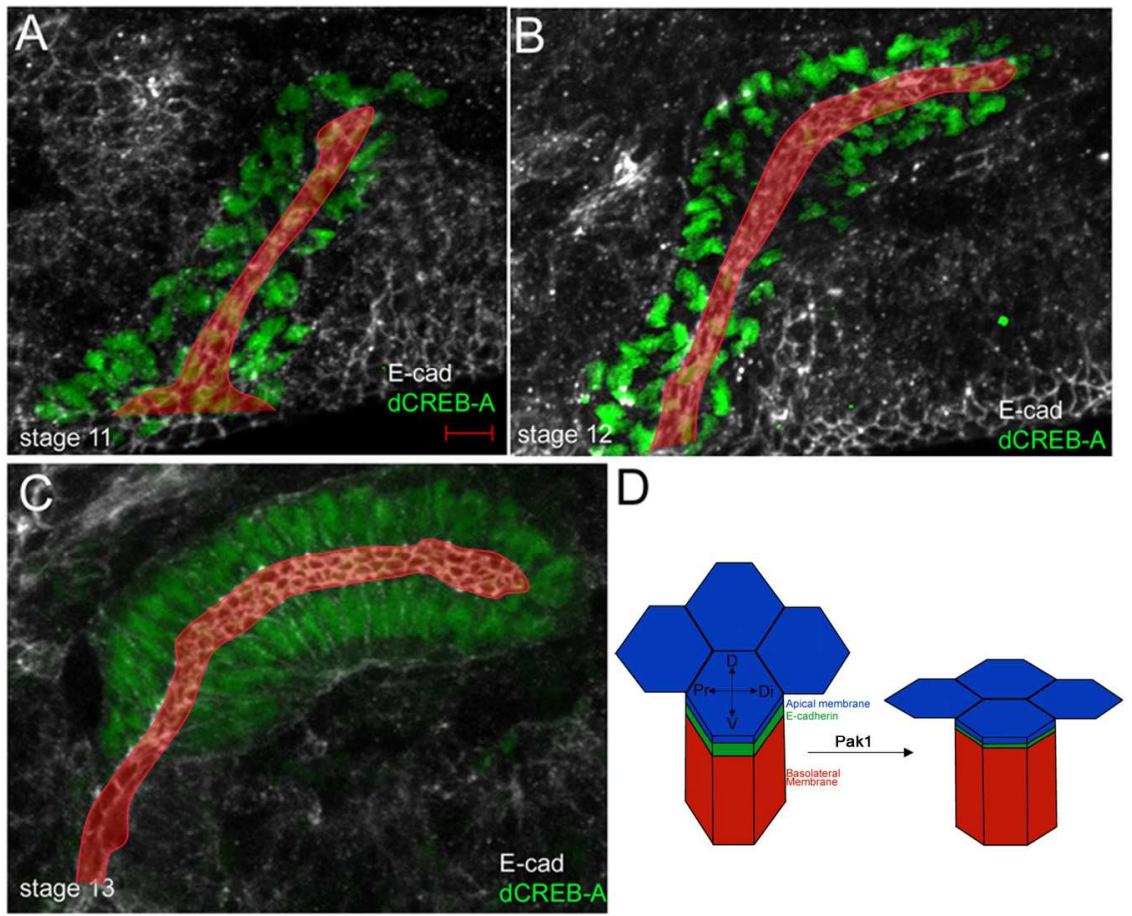

Fig. 2. Lumen elongation in the Drosophila embryonic salivary gland.

(A) The salivary gland lumen is formed as salivary gland cells invaginate from the embryo surface at stage 11. (B and C) As the gland migrates posteriorly, lumen length increases. (D) Apical domain elongation is controlled by differential localization of E-cadherin in a Pak1-dependent manner. Panels in A-C are projected confocal images of wild-type embryos stained for dCREB-A (green) to mark the gland nuclei and E-cadherin (E-cad; white) to mark the gland lumen that is outlined in red. Scale bar in A represents $5 \mu \mathrm{m}$.

the lumens that form are expanded or branched (Myat and Andrew, 2002). Similar to loss of hairy function, overexpression of $h k b$ in salivary gland cells leads to expanded and rounded lumens, instead of elongated lumens, but not branched lumens (Myat and Andrew, 2002). $\mathrm{Hkb}$ controls salivary gland lumen size through two downstream target genes, klarsicht (klar), which encodes a Drosophila KASH (Klar, Anc-1, Syne-1 homology) domain protein (Mosley-Bishop et al., 1999), and crumbs ( $\mathrm{rrb}$ ), which encodes an apical transmembrane protein that confers apical identity (Myat and Andrew, 2002; Wodarz et al., 1995). In Drosophila ovaries and eye, Klar is present on the nuclear envelope and is required for nuclear migration whereas in the early embryo, Klar localizes to lipid droplets and is required for lipid droplet transport (Guo et al., 2005; Kracklauer et al., 2007; Welte et al., 1998). Similar to Klar, the mammalian KASH domain proteins, such as Nesprins, localize to nuclear membranes (Zhang et al., 2001) and regulate nuclear positioning (Zhang et al., 2010). Crumbs $(\mathrm{Crb})$ is important for the establishment and maintenance of apical polarity in both Drosophila and mammalian epithelia and photoreceptor cells (Bulgakova and Knust, 2009; Izaddoost et al., 2002; Pellikka et al., 2002; Tepass et al., 1996; Tepass and Knust, 1990; 
Wodarz et al., 1995). Hkb regulates not only the levels of $c r b$ RNA in salivary gland cells but also Crb protein level and/or localization together with Klar (Myat and Andrew, 2002). Considering Klar likely mediates dynein-dependent cargo transport along microtubules (Mosley-Bishop et al., 1999; Welte et al., 1998), it is thought that Hkb mediates the apical delivery of vesicles, such as those containing $\mathrm{Crb}$, through Klar, to promote apical membrane growth and polarized elongation of apical domains during salivary gland lumen elongation.

\subsection{Ribbon function in salivary gland lumen elongation}

crb RNA expression in salivary gland cells is also controlled by Ribbon (Rib), a BTB (brica-brac, tramtrack, broad-complex)/POZ (poxvirus and zinc finger) domain transcription factor required for the proper morphology of multiple tubular organs in Drosophila, such as the salivary gland, trachea, Malpighian tubules and the hindgut (Blake et al., 1998; Bradley and Andrew, 2001; Jack and Myette, 1997; Kerman et al., 2008; Shim et al., 2001). In salivary gland cells, Rib controls lumen elongation by simultaneously promoting $c r b$ RNA expression and limiting apical localization of active phosphorylated Moesin (Moe), a Drosophila Ezrin-Radixin-Moesin (ERM) family protein that links the actin cytoskeleton to the plasma membrane (Kerman et al., 2008). The salivary gland phenotype of rib mutants is phenocopied by gland specific expression of Moe ${ }^{T 559 D}$, a phosphomimetic mutation in Moe where threonine (T) 559 is replaced by aspartic acid (D) and functions as a constitutively-active form of Moe in Drosophila developing eyes (Karagiosis and Ready, 2004; Kerman et al., 2008). Since Moe normally links the actin cytoskeleton to the plasma membrane, it is thought that inhibition of Moe activity by Rib decreases the linkage of the apical membrane to the actin cytoskeleton, which in turn, reduces apical membrane stiffness to allow lumen elongation. Furthermore, Rib may control lumen elongation by promoting Rab11-dependent delivery of apically targeted vesicles since rib mutant gland cells have a reduced number of apical Rab-11 positive vesicles (Kerman et al., 2008). Rab11 is a small GTPase that mediates apical trafficking of cargo proteins through recycling endosomes or directly from the Golgi (Satoh et al., 2005). Based on these observations, a model for Rib regulation of salivary gland lumen elongation is proposed where Rib promotes crb RNA expression and Rab11-dependent apical vesicle delivery to facilitate apical membrane growth, and limits apical Moe activity to reduce apical membrane stiffness which allows the salivary gland lumen to elongate (Kerman et al., 2008). This model is supported by computational models based on live imaging, which suggest that rib mutant salivary glands have increased apical stiffness and apical viscosity compared to wild-type salivary glands (Cheshire et al., 2008).

\subsection{Pak1 is required for correct salivary gland lumen width}

Recent studies from our laboratory demonstrate an essential role for the p21 activated kinase (Pak) 1 in control of salivary gland lumen size through the cell-cell adhesion protein, E-cadherin. Pak proteins are serine-threonine kinases that control vascular integrity in zebrafish blood vessels (Buchner et al., 2007; Liu et al., 2007) and lumen formation by human endothelial cells cultured in three-dimensional collagen matrices (Koh et al., 2008; Koh et al., 2009). In the Drosophila embryonic salivary gland, Pak1 functions downstream of the small 
GTPase, Cdc42, to regulate gland lumen size (Pirraglia et al., 2010). Loss of pak1 results in expansion of lumen diameter in the medial and distal regions of the gland without affecting lumen length. The widened lumen of pak1 mutant salivary glands is not due to increased cell proliferation, and instead, is due to failure to limit apical domain size and to elongate the apical domain in the direction of lumen elongation. These changes in apical domain size and elongation in pak1 mutant gland cells is accompanied by increased localization of E-cadherin, at the adherens junctions (AJs) and reduced localization at the basolateral membrane (Figure 2D). Pak1 controls this differential localization of E-cadherin in salivary gland cells through Rab5- and Dynamin-dependent endocytosis; not only does inhibition of either Rab5 or Dynamin in salivary gland cells phenocopy the pak1 mutant lumen defects, but expression of constitutively-active Rab5 in pak1 mutant gland cells restores normal distribution of E-cadherin and restores normal apical domain size and elongation (Pirraglia et al., 2010). Pak1 may regulate E-cadherin endocytosis indirectly through its downstream effector Merlin, the Drosophila homologue of the human neurofibromatosis 2 gene (McClatchey and Fehon, 2009 ), since expression of dominant-negative Merlin phenocopies the salivary gland lumen defects of pak1 and Rab5 mutant embryos. Thus, Pak1-dependent localization of E-cadherin at the AJs and at the basolateral membrane is important for apical domain elongation and control of salivary gland lumen size (Figure 2D). A role for Pak1 in lumen size control through membrane transport of E-cadherin is further supported by the demonstration that expression of an activated membrane-bound form of Pak1 in the salivary gland forms multiple intercellular lumens instead of a single central lumen. Induction of multiple intercellular lumens by activated Pak1 is due to the internalization of E-cadherin and apical membrane proteins into early endosomes (Pirraglia et al., 2010).

\subsection{Control of salivary gland lumen size through secretory activity}

While dynamic changes at the apical membrane and differential localization of E-cadherin control salivary gland lumen size early in gland development, directed secretion into the lumenal space expands lumen width and allows formation of a patent lumen in the mature gland. Secretory products are detected as electron dense material by TEM within apical vesicular structures and in the luminal space. As embryogenesis proceeds, the salivary gland lumen continues to fill with electron-dense secreted products and lumen width increases uniformly throughout the length of the lumen (Myat and Andrew, 2002; Seshaiah et al., 2001). Secretory function of salivary gland cells is controlled by pasilla (ps) which encodes a Drosophila homologue of the human Nova family RNA-binding proteins that function in RNA splicing (Jensen et al., 2000; Seshaiah et al., 2001), and by PH4aSG1 and PH4aSG2, which encode homologues of the a-subunit of resident endoplasmic reticulum enzymes that hydroxylate proline in select secreted proteins (Abrams et al., 2006; Kivirikko and Pihlajaniemi, 1998 ). In $p s$ mutant salivary glands, secretory contents within the lumen and apical vesicles is reduced, and the lumen fails to expand uniformly (Seshaiah et al., 2001). Similar to $p s$ mutant embryos, PH4aSG1 and PH4aSG2 mutant embryos have reduced secretory products in the salivary gland lumens and are characterized by abnormally shaped lumens with regions of expansion, constriction and closure (Abrams et al., 2006). Together these studies show that ps, PH4aSG1 and PH4aSG2 control salivary gland lumen size at later stages of embryogenesis by affecting secretion into the gland lumen. 
The expression of $P H 4 a S G 1$ and $P H 4 a S G 2$ is regulated by the single Drosophila FoxA family transcription factor Fork head (Fkh), that affects 59\% of gene expression in the salivary gland (Maruyama et al., 2011) and is required for cell survival and cell shape change during salivary gland invagination (Myat and Andrew, 2000b). Fkh regulates the expression of sage, encoding a salivary gland specific basic helix-loop-helix (bHLH) protein, and functions with Sage to directly regulate the expression of PH4aSG2 and to indirectly regulate the expression of PH4aSG1 (Abrams et al., 2006). In addition to Fkh, secretory activity in the Drosophila salivary glands is controlled by CrebA which belongs to the CrebA/Creb3-like family of bZip transcription factors (Abrams and Andrew, 2005; Andrew et al., 1997; Fox et al., 2010). CrebA can bind directly to the enhancers of genes encoding components in secretory pathways and upregulate the expression of genes encoding both the general protein machinery required for secretion and of cell type-specific secreted proteins (Fox et al., 2010). Consistent with the role of CrebA in salivary gland secretion, lumens of CrebA mutant embryos are smaller and have reduced secretory material (Fox et al., 2010).

In summary, salivary gland lumen size in early stages of gland development is controlled by apical membrane growth and apical domain elongation in individual gland cells through processes regulated by transcription factors, Hairy, Hkb and Rib, and their downstream targets, Klar, Crb and Moe as well as by Cdc42 and its effector Pak1 through differential localization of E-cadherin. During late embryogenesis, uniform expansion of the gland lumen is controlled by directed secretion into the lumen through the activities of $p \mathrm{~s}$, PH4aSG1, PH4aSG2 and CrebA.

\section{Trachea}

The Drosophila trachea serves as the respiratory organ of the animal, and like the vertebrate lung, salivary gland and vasculature it is a branched network of tubes. The pattern of the larval trachea is established during embryogenesis when cells from ten tracheal placodes or plates of approximately 90 ectodermal epithelial cells on each side of the embryo, invaginate into the underlying mesoderm to form elongated sacs (Figure 3A). In response to Fibroblast Growth Factor (FGF) or Branchless (Bnl), which is expressed in surrounding ectodermal and mesodermal cells (Ohshiro et al., 2002; Sutherland et al., 1996; Zhan et al., 2010), the invaginated tracheal cells which express the FGF receptor, Breathless (Btl), migrate towards the $\mathrm{Bnl}$ source to form the six primary branches (Figure 3B and C). Some of the primary branches, such as the visceral branch (VB) and the anterior and posterior dorsal trunk (DT), grow along the anterior-posterior axis, whereas other branches, such as the dorsal branch (DB), lateral trunk (LT) and ganglionic branch (GB), grow along the dorsal-ventral axis (Figure 3D). Tracheal cell migration is followed by fusion between the contralateral DBs, DT and LT branches of adjacent segmentally arranged metameres on each side of the embryo to form an interconnected tracheal network with a single central lumen (Figure 3F).

Similar to the Drosophila embryonic salivary gland, the lumen of the trachea is formed during the invagination step when cells of the placode become internalized and form elongated sacs (Casanova, 2007). As the internalized tracheal cells migrate out to form the six primary branches, the lumen extends simultaneously with the elongating branches. In this section, we focus on how lumen size is controlled in the trachea and how lumens form de novo at two distinct stages of tracheal development, first, during anastomosis of the tracheal DT, and second, during intracellular lumen formation in the specialized terminal cells. 

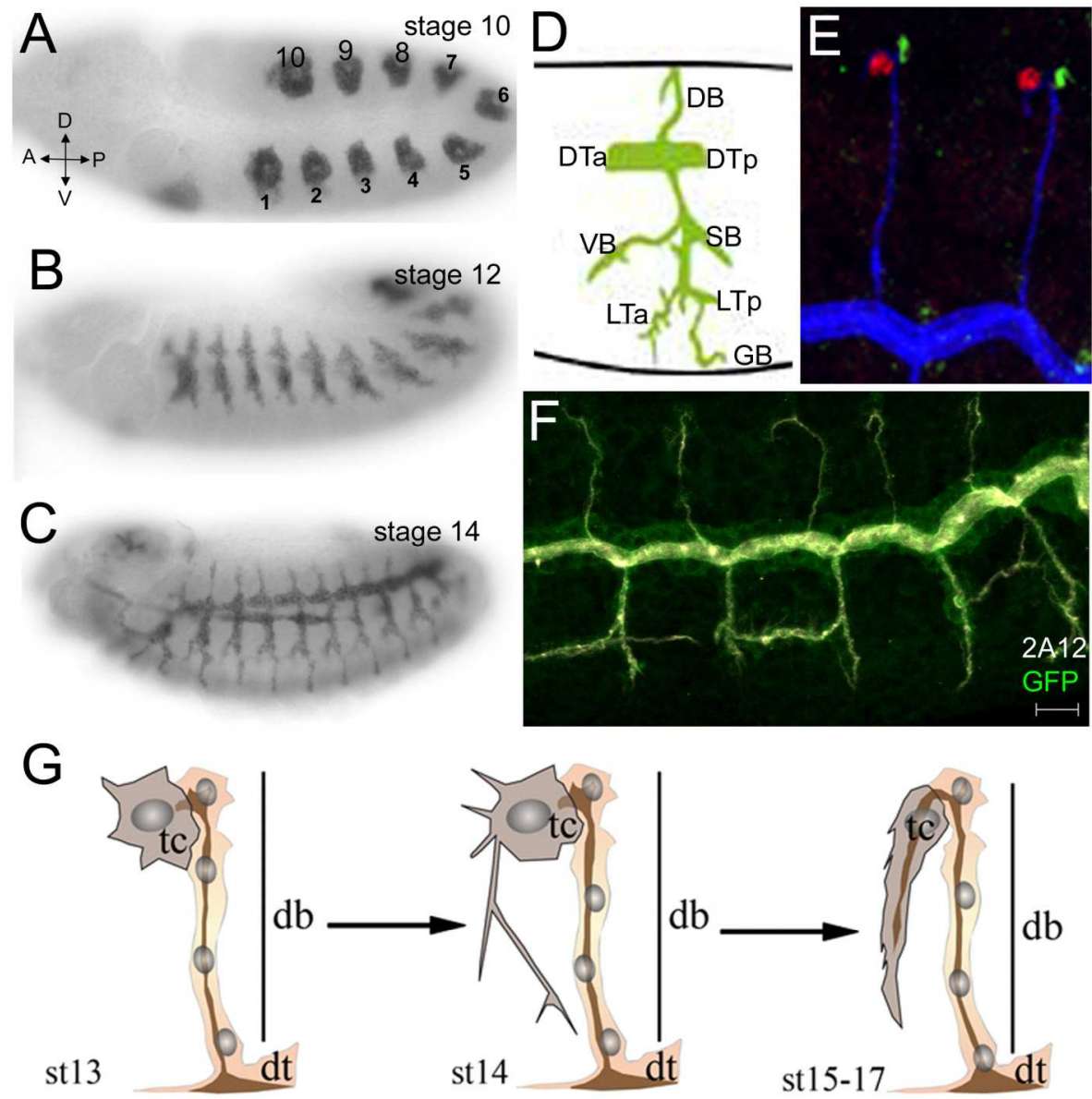

Fig. 3. Lumen formation in the tracheal branches.

(A) The embryonic trachea is formed from 10 placodes of ectodermal cells that invaginate into the interior of the embryo. D: dorsal; V: ventral; A: anterior and P: posterior. (B) Internalized tracheal cells migrate out to form the six primary branches, some of which fuse with branches from adjacent metameres to form an interconnected network (C). (D) Schematic diagram of one tracheal metamere showing the dorsal trunk (anterior and posterior; Dta and Dtp), lateral trunk (anterior and posterior; Lta and Ltp), dorsal branch (DB), visceral branch (VB), spiracular branch (SB) and ganglionic branch (GB). (E) Two DBs, each with a terminal cell (TC, red) and a fusion cell (FC, green). (F) The interconnected tracheal network of a stage 15 embryo has a single central lumen. (G) Terminal cell (TC) forms a lumen de novo as the cell elongates; db: dorsal branch, dt: dorsal trunk. Embryos in A-C were processed for RNA in situ hybridization to trachealess to label tracheal cells. Embryo in E was stained for DSRF (red) to label TC, Dysfusion to label FC (green) and 2A12 (blue) to label the lumen of the DB. Embryo in F was stained for 2A12 (white) to label the lumen and GFP to detect actin-GFP expressed specifically in the trachea with breathless-GAL4. Diagram in D is not drawn to scale. Panel G was kindly provided by J. Casanova with permission from the Nature Publishing Group. 


\subsection{Regulation of tracheal tube/lumen size and shape}

Morphometric and genetic analyses in the Drosophila embryonic trachea were among the first to show that tube and lumen size are under genetic control. Tracheal tube length increases gradually, whereas tube diameter increases abruptly at distinct times during development. By the larval stage, tracheal tube diameter can be 40x times its original size (Beitel and Krasnow, 2000). These morphometric studies by Beitel and Krasnow (2000) were the first to show that tracheal tube size is not controlled by the number, size or shape of the cells that comprise the tube, and instead, is controlled at the apical surface of the tracheal cells and by the overall identity of each branch. The role of the apical surface membrane in control of tracheal tube size is supported by studies of the grainy head ( $g r h)$ mutant (Hemphala et al., 2002). In grh mutant embryos, tracheal DT length is increased by $40 \%$ and is characterized by the dramatic growth of the apical surface membrane. Grh encodes a transcription factor that is expressed in a number of epithelial tissues (Bray and Kafatos, 1991; Ostrowski et al., 2002), including the epidermis, where Grh controls re-epithelialization during wound healing through the tyrosine kinase Stitcher (Wang et al., 2009). In the trachea, Grh acts downstream of Bnl/FGF signaling to limit lumen elongation and thus, ensure that branches with lumens of the correct size are formed (Hemphala et al., 2002).

A second mechanism by which tracheal lumen size is controlled is through the luminal secretion and modification of chitin, a fibrous substance composed of polysaccharides. Secretion of chitin occurs prior to expansion of the DT lumen and continues throughout growth of the DT lumen. Transient accumulation of chitin is thought to coordinate and stabilize expansion of the lumen (Araujo et al., 2005; Devine et al., 2005; Tonning et al., 2005). While genes encoding proteins that synthesize and secrete chitin control the uniform expansion of tracheal lumen diameter and elongation of lumen length, genes encoding proteins that modify chitin, specifically control lumen length. In mutants where chitin fibers do not form, the DT lumen at points of anastomosis between branches of neighboring hemisegments fail to expand, whereas the lumen throughout the rest of the DT is excessively dilated (Moussian et al., 2006; Tonning et al., 2005). By contrast, in mutants for Vermiform (Verm) and Serpentine (Serp), which encode chitin deacetylating proteins, the DT lumen is excessively elongated (Luschnig et al., 2006; Wang et al., 2006). Restriction of tracheal tube length also depends on genes encoding components of the SJs, a structure located basal to the adherens junctions, which like the vertebrate tight junctions functions as a paracellular diffusion barrier and is comprised in part by the claudin family proteins (Behr et al., 2003; Nelson et al., 2010; Tepass et al., 2001; Wu et al., 2004). Mutations in several SJ proteins affect both tracheal tube length and diameter but not early aspects of tracheal development (Behr et al., 2003; Beitel and Kransnow, 2000; Llimargas et al., 2004; Paul et al., 2003; $\mathrm{Wu}$ et al., 2004). One mechanism by which SJ proteins control tracheal tube size is through apical secretion of Verm and Serp (Luschnig et al., 2006; Wang et al., 2006). SJassociated polarity proteins, such as Discs Large (Dlg) and Scribble (Scrib), also control tracheal tube length independent of chitin and without affecting the paracellular diffusion barrier function (Laprise et al., 2010). The FERM domain protein, Yurt, which belongs to the Yurt/Coracle group of basolateral polarity proteins controls tracheal lumen length by antagonizing the apical determinant protein, Crumbs. However, unlike Yurt, Scrib controls lumen size independent of Crb. The SJ-associated protein, Coracle (Cora), regulates tracheal lumen length by limiting Crb activity independent of Yrt as well as by promoting Verm secretion (Laprise et al., 2010). Thus, independent of Verm/Serp-dependent chitin 
modification, tracheal lumen length is controlled by a Yurt/Cora pathway dependent on $\mathrm{Crb}$ activity, and by a Scrib pathway independent of Crb. SJs likely control tracheal tube size by other mechanisms, such as cell shape since mutations in the SJ proteins, encoded by megatrachea and lachesin, cause tracheal cells to adopt an irregularly stretched morphology (Behr et al., 2003; Llimargas et al., 2004).

Apical secretion of luminal contents precedes expansion of tube diameter and occurs in a sudden burst through COPI-, COPII- and Sec24-dependent membrane transport (Forster et al., 2010; Grieder et al., 2008; Jayaram et al., 2008; Tsarouhas et al., 2007) and is dependent on RhoDiaphanous-Myosin V transport (Massarwa et al., 2009). In addition to secretion of chitin, apical secretion may also play a role in tube length by contributing to apical membrane growth and/or targeting other as of yet unidentified, regulators of tube size to the luminal surface. At the end of embryogenesis, the chitin scaffold that has served to control lumen size in the trachea is removed in time for larval hatching when the trachea gets filled with oxygen and other gases. This is achieved through an endocytic pulse that allows the tracheal cells to internalize and clear away the luminal contents (Tsarouhas et al., 2007). The small GTPases, Rab5 and Dynamin are required for the endocytic pulse and luminal protein clearance (Tsarouhas et al., 2007). These studies highlight the important role that chitin plays in tracheal lumen size control; however, it is not known how chitin fibers allow uniform diametric growth of the tracheal tube and restrict tube length. Independent of chitin and SJs, tracheal lumen size is likely to be controlled by additional mechanisms, such as that demonstrated by Convoluted/dALS (Swanson et al., 2009) and by serrano mutants which implicate the planar cell polarity pathway in control of tracheal tube length (Chung et al., 2009).

Lumen shape in the tracheal tubes is controlled by receptor tyrosine phosphatases. In embryos double mutant for two receptor-linked protein-tyrosine phosphatases (RPTPs), Ptp4E and Ptp10D, tracheal branches, such as the ganglionic branches and terminal branches, form large bubble-like cysts with dilated lumens that stain positive for apical marker proteins (Jeon and Zinn, 2009). Cyst size and number are increased upon expression of activated Egfr (epidermal growth factor receptor) and decreased with reduction of Egfr. Thus, proper lumen shape in the trachea is achieved through downregulation of Egfr signaling by the Ptp4E and Ptp10D RPTPs.

\subsection{Dorsal trunk anastomosis}

During primary branch outgrowth, the tracheal lumen is initially closed at the branch tips. Later in development, a continuous tubular network is formed during anastomosis, when specialized cells, known as fusion cells, which are found at the tips of migrating branches such as the DT and DB, recognize each other's partner in the adjacent metamere and connect to form a continuous lumen (Figure 3E) (Baer et al., 2009). Although these specialized cells are called fusion cells, they, in fact, do not fuse themselves and instead mediate the fusion of two separate tubular structures. The tracheal fusion process occurs in four distinct steps. In the first step, tracheal cells at the tip of adjacent branches contact each other through filopodial extensions. In the second step, fusion cells form a cytoskeletal track at the site of contact consisting of F-actin, microtubules, the plakin Short Stop (Shot) and E-cadherinbased adhesion complexes that are assembled de novo at the contact site (Lee and Kolodziej, 2002). Structure function studies showed that distinct sites within the cytoplasmic domain of E-cadherin control the initial assembly of the F-actin track, recruitment of Shot and subsequent maturation of the track in a microtubule-dependent manner (Lee et al., 2003). In 
the third step, the cytoskeletal track expands to span the fusion cells and bridge the apical surfaces of the DT lumens with apical membrane formed de novo at the contact site. In the fourth and final step, the cytoskeletal track disassembles, the apical surfaces become continuous and the narrow lumen that is initially formed expands to its final size (Baer et al., 2009). Connection of the pre-existing DT lumens to the new lumen is dependent on targeted exocytosis and remodeling of the plasma membrane by the Arf-like 3 small GTPase (Arl3) which is known to associate with microtubules and vesicles (Jiang et al., 2007; Kakihara et al., 2008), and the COPI coatomer complex that mediates membrane transport of small vesicles (Grieder et al., 2008). Therefore, tracheal branch fusion is a complex and highly regulated process involving precise coordination of cytoskeletal proteins, adhesion proteins and components of the vesicular trafficking machinery.

\subsection{Terminal cell lumen formation}

Terminal cells (TCs) at the tips of some tracheal branches form intracellular lumens de novo (Figure 3E and G). Although de novo lumen formation in TCs was initially thought to occur by the "cell hollowing" mechanism (Lubarsky and Krasnow, 2003), recent studies by Gervais and Casanova (2010) show that the intracellular lumen forms by the inward growth of new apical membrane from the surface that is in contact with the adjacent tracheal cell and not through a cell-hollowing mechanism, shedding significant insight into this process. The TC elongates as its lumen is formed intracellularly and both these processes are accompanied by the asymmetric accumulation of the actin and microtubule cytoskeletal systems (Figure 3G). Genetic perturbation of the microtubule network results in defects in TC lumen elongation suggesting a critical role for microtubules in TC lumen formation. The Bnl/FGF signaling pathway, known to regulate multiple aspects of tracheal morphogenesis as described above, also regulates TC lumen elongation; in embryos with reduced gene dosage of $b n l$, TC lumen length is shortened (Zhan et al., 2010). Bnl/FGF signaling controls TC elongation and intracellular lumen formation by regulating actin and microtubules through Drosophila Serum Response Factor (DSRF) and Enabled, a VASP protein (Gervais and Casanova, 2010); however, DSRF is not required for Bnl-dependent initiation of TC elongation and lumen formation, and instead allows these processes to progress under normal conditions (Gervais and Casanova, 2011).

In addition to the requirement of Bnl signaling for intiation and progression of TC elongation and lumen formation, integrin-mediated adhesion between the terminal branches and the surrounding extracellular matrix is necessary for maintaining these tubes and for proper organization of the intracellular lumen (Levi et al., 2006). The amenability of Drosophila to large-scale genetic screens has allowed the generation of many new mutants affecting TC lumen formation (Ghabrial et al., 2011). Analysis of these new mutants is bound to bring novel insights to lumen formation in the tracheal TCs in the years to come.

\section{Conclusion}

In this chapter, we have reviewed our current understanding of how lumens form and are maintained in the dorsal vessel, salivary gland and trachea of the Drosophila embryo. Lumen formation in the Drosophila embryonic salivary gland and primary branches of the trachea occurs concomitantly with invagination of the salivary gland and tracheal cells from the embryo surface. Thus, it is not entirely surprising that lumen size control in these two epithelial-based organs share similar cellular and molecular mechanisms, such as the roles 
of apical membrane growth and luminal secretion in defining tube and lumen size. Although a role for chitin fibers in uniform diametric growth and restriction of tube length has not been documented for the salivary gland as in the trachea, evidence does exist for luminal secretion in forming a patent lumen in the salivary gland.

The dorsal vessel forms by entirely distinct mechanisms from that of the salivary gland and trachea; however, in terms of lumen formation and size control, there are conserved mechanisms between the dorsal vessel and salivary gland and trachea. For example, Ecadherin-mediated cell-cell adhesion is important for correct lumen size in the salivary gland and for forming a luminal space between CBs in the dorsal vessel. Although endocytic trafficking of E-cadherin is important for lumen size control in the salivary gland, it is not known whether Slit/Robo inhibition of E-cadherin occurs by a similar or distinct mechanism. In addition to E-cadherin, SJ proteins, such as Coracle, are required for correct lumen size in the trachea and the dorsal vessel. In the trachea, it is well established that synthesis, secretion and modification of chitin affects tube and lumen size. Although dorsal vessel shape is affected in SJ mutants, it is not known whether vessel diameter and/or length are affected as well. It was recently reported that the lumen of the mouse dorsal aorta forms by a "cord-hollowing" mechanism where lumen formation between two cells is initiated extracellularly through repositioning of cadherin-based adherens junctions and through repulsion of apposed lateral membranes (Lubarsky and Krasnow, 2003; Strillic et al., 2009). Due to the similarities between lumen formation in the Drosophila dorsal vessel and the mouse aorta, studies of lumen formation in the Drosophila dorsal vessel will continue to yield insight into lumen formation in the vertebrate vasculature.

Studies in the Drosophila embryonic salivary gland showed that growth of the apical membrane and modulation of E-cadherin localization at the adherens junctions and the basolateral membrane can influence lumen size and number. In the Drosophila embryonic salivary gland, the regulated process of invagination ensures that only a single central lumen is formed; however, the single central lumen can be replaced by multiple intercellular lumens, such as by expression of activated Pak1, as described above. By contrast, the formation of multiple lumens is a normal intermediate step in the formation of a single central lumen during zebrafish gut tube morphogenesis (Bagnat et al., 2007) and in pathological conditions, such as pre-invasive breast cancer, where multiple lumens characterize cribriform ductal carcinoma in situ (DCIS) (Jaffar and Bleiweiss, 2002). Thus, understanding the mechanisms by which tubular organs can transition between single and multiple lumens will increase our understanding of more complex processes, such as DCIS.

In the Drosophila embryonic trachea, de novo lumen formation occurs during anastomosis of specific branches, such as the DT, between adjacent hemisegments. A similar process of de novo lumen formation occurs during anastomosis of vascular sprouts during angiogenesis. Like the tracheal tip cells of the Drosophila trachea, vascular tip cells extend filopodia to explore the surrounding enrivonment. Moreover, the presence of vascular E-cadherin (VEcadherin) at the tips of filopodia in cultured human endothelial cells (Almagro et al., 2010) and at tip-tip contact sites between neighboring sprouts during formation of the zebrafish intersegmental vessel (Blum et al., 2008) suggest a role for VE-cadherin in vascular anastomosis that parallels the role played by E-cadherin in Drosophila tracheal anastomosis.

Although the structure and function of the Drosophila embryonic dorsal vessel, salivary gland and trachea may differ from more complex organs of other organisms, it is clear that there are conserved mechanisms for lumen formation. Thus, the study of tube and lumen 
formation in Drosophila tubular organs will continue to yield novel mechanisms and shed significant insight into how lumens form in more complex organisms.

\section{Acknowledgements}

We are grateful to F. Macabenta and S. Kramer for providing Figure 1C and for critical reading of the manuscript. We also thank J. Casanova for providing Figure 3G.

\section{References}

Abrams, E. \& Andrew, D. (2005). CrebA regulates secretory activity in the Drosophila salivary gland and epidermis. Development 132, 2743-2758.

Abrams, E., Milhoulides, W. \& Andrew, D. (2006). Fork head and Sage maintain a uniform and patent salivary galnd lumen through regulation of two downstream target genes, PH4alphaSG1 and PH4alphaSG2. Development 133, 3517-3527.

Albrecht, S., Altenhein, B. \& Paululat, A. (2011). The transmembrane receptor Uncoordinated5 (Unc5) is essential for heart lumen formation in Drosophila melanogaster. Developmental Biology 350 89-100.

Almagro, S., Durmort, C., Chervin-Petinot, A., Heyraud, S., Dubois, M., Lambert, O., Maillefaud, C., Hewat, E., Schaal, J., Huber, P. et al. (2010). The motor protein myosin-X transports VE-cadherin along filopodia to allow the formation of early endothelial cell-cell contacts. Mol Cell Biol 30, 1703-1717.

Andrew, D., Baig, A., Bhanot, P., Smolik, S. \& Henderson, K. (1997). The Drosophila dCrebA gene is required for dorsal/ventral patterning of the larval cuticle. Development, 181-193.

Andrew, D. \& Ewald, A. (2010). Morphogenesis of epithelial tubes: Insights into tube formation, elongation and elaboration. Dev Biol 341, 34-55.

Araujo, S., Aslam, H., Tear, G. \& Casanova, J. (2005). mummy/cystic encodes an enzyme required for chitin and glycan synthesis, involved in trachea, embryonic cuticle and CNS development - Analysis of its role in Drosophila tracheal morphogenesis. Developmental Biology 288, 179-193.

Baer, M. M., Chanut-Delalande, H. \& Affolter, M. (2009). Cellular and Molecular Mechanisms Underlying the Formation of Biological Tubes. Current Topics in Developmental Biology 89, 137-162.

Bagnat, M., Cheung, I., Mostov, K. \& Stainier, D. (2007). Genetic control of single lumen formation in the zebrafish gut. Nat Cell Biol 954-960.

Banerjee, S., Sousa, A. \& Bhat, M. (2006). Organization and function of septate junctions: an evolutionary perspective. Cell Biochem Biophys 46, 65-77.

Behr, M., Riedel, D. \& Schuh, R. (2003). The Claudin-like Megatrachea is essential in septate junctions for the epithelial barrier function in Drosophila. Developmental Cell 5, 611-620.

Beitel, G. \& Krasnow, M. A. (2000). Genetic control of epithelial tube size in the Drosophila tracheal system. Development 127, 3271-3282.

Blake, K., Myette, G. \& Jack, J. (1998). The products of ribbon and raw are necessary for proper cell shape and cellular localization of nonmuscle myosin in Drosophila. Dev Biol 147, 177-88.

Blum, Y., Belting, H., Ellertsdottir, E., Herwig, L., Luders, F. \& Affolter, M. (2008). Complex cell rearrangements during intersegmental vessel sprouting and vessel fusion in the zebrafish embryo. Dev Biol 316, 312-322. 
Bradley, P. B. \& Andrew, D. J. (2001). Ribbon encodes a novel BTB/POZ protein required for directed cell migration in Drosophila melanogaster. Development 128, 3001-3015.

Bray, S. \& Kafatos, F. (1991). Developmental function of Elf-1: an essential transcription factor during embryogenesis in Drosophila. Genes Dev 5, 1672-1683.

Buchner, D., Su, F., Yamaoka, J., Kamei, M., Shavit, J., Barthel, L., McGee, B., Amigo, J., Kim, S., Hanosh, A. et al. (2007). pak2a mutations cause cerebral hemorrhage in redhead zebrafish. PNAS 104, 13996-14001.

Bulgakova, N. \& Knust, E. (2009). The Crumbs complex: from epithelial cell-polarity to retinal degeneration. J Cell Sci 122, 2587-2596.

Carroll, S., Laughon, A. \& Thalley, B. (1988). Expression, function and regulation of the hairy segmentation protein in the Drosophila embryo. Genes and Development 2, 883-890.

Casanova, J. (2007). The emergence of shape: notions from the study of the Drosophila tracheal system. EMBO Rep. 8, 335-339.

Chanana, B., Steigemann, P., Jackle, H. \& Vorbruggen, G. (2009). Reception of Slit requires only the chondroitin-sulphate-modified extracellular domain of Syndecan at the target cell surface. PNAS 106, 11984-11988.

Chartier, A., Zaffran, S., Astier, M., Semeriva, M. \& Gratecos, D. (2002). Pericardin, a Drosophila type IV collagen-like protein is involved in the morphogenesis and maintenance of the heart epithelium during dorsal ectoderm closure. Development 129, 3241-3253.

Cheshire, A., Kerman, B., Zipfel, W., Spector, A. \& Andrew, D. (2008). Kinetic and mechanical analysis of live tube morphogenesis. Developmental Dynamics, 2874-2888.

Chung, S., Vining, M., Bradley, P., Chan, C., Wharton, K. J. \& Andrew, D. (2009). Serrano (sano) functions with the planar cell polarity genes to control tracheal tube length. PLoS Genet 5, e1000746.

Devine, P., Lubarsky, B., Shaw, K., Luschnig, S., Messina, L. \& Krasnow, M. A. (2005). Requirement for chitin biosynthesis in epithelial tube morphogenesis. PNAS 102, 17014-17019.

Forster, D., Armbruster, K. \& Luschnig, S. (2010). Sec24-dependent secretion drives cellautonomous expansion of tracheal tubes in Drosophila. Current Biology 20, 62-68.

Fox, R., Hanlon, C. \& Andrew, D. (2010). The CrebA/Creb-like transcription factors are major and direct regulators of secretory capacity. J Cell Biol 191, 479-92.

Gervais, L. \& Casanova, J. (2010). In vivo coupling of cell elongation and lumen formation in a single cell. Current Biology 20, 359-366.

Gervais, L. \& Casanova, J. (2011). The Drosophila homologue of SRF acts as a boosting mechanism to sustain FGF-induced terminal branching in the tracheal system. Development 138, 1269-1274.

Ghabrial, A., Levi, B. \& Krasnow, M. (2011). A systematic screen for tube morphogenesis and branching genes in the Drosophila tracheal system. PLoS Genet 7, e1002087.

Grieder, N. C., Caussinus, E., Parker, D. S., Cadigan, K. \& Affolter, M. (2008). $\gamma$ COP is required for apical secretion and epithelial morphogenesis in Drosophila melanogaster. PLoS One 3, e3241.

Guo, Y., Jangi, S. \& Welte, M. (2005). Organelle-specific control of intracellular transport: distinctly targeted isoforms of the regulator Klar. Mol Biol Cell. 16, 1406-1416.

Haag, T., Haag, N., Lekven, A. \& Hartenstein, V. (1999). The Role of Cell Adhesion Molecules in Drosophila Heart Morphogenesis: Faint Sausage, Shotgun/DECadherin, and Laminin A Are Required for Discrete Stages in Heart Development. Developmental Biology 208, 56-69. 
Hemphala, J., Uv, A., Cantera, R., Bray, S. \& Samakovlis, C. (2002). Grainy head controls apical membrane growth and tube elongation in response to Branchless/FGF signaling. Development 130, 249-258.

Hooper, K. L., Parkhurst, S. M. \& Ish-Horowicz, D. (1989). Spatial control of hairy protein expression during embryogenesis. Development 107, 489-504.

Izaddoost, S., Nam, S. C., Bhat, M. A., Bellen, H. J. \& Choi, K. W. (2002). Drosophila crumbs is a positional cue in photoreceptor adherens junctions and rhabdomeres. Nature 416, 178-183.

Jack, J. \& Myette, G. (1997). The genes raw and ribbon are required for proper shape of tubular epithelial tissues in Drosophila. Genetics 147, 243-253.

Jaffar, S. \& Bleiweiss, I. (2002). Histologic classification of ductal carcinoma in situ. Microscopy research and technique 59, 92-101.

Jayaram, S., Senti, K., Tiklova, K., Tsahouras, V., Hemphala, J. \& Samakovlis, C. (2008). COPI vesicle transport is a common requirement for tube expansion in Drosophila. PLoS One 3, e1964.

Jensen, K., Dredge, B., Stefani, G., Zhong, R., Buckanovich, R., Okano, H., Yang, Y. \& Darnell, R. (2000). Nova-1 regulates neuron-specific alternative splicing and is essential for neuronal specificity. Neuron 25, 359-371.

Jeon, M. \& Zinn, K. (2009). Receptor tyrosine phosphatases control tracheal tube geometries through negative regulation of Egfr signaling. Development 136, 3121-3129.

Jiang, L., Rogers, S. L. \& Crews, S. T. (2007). The Drosophila dead end Arf-like 3 GTPase controls vesicle trafficking during tracheal fusion cell morphogenesis. Dev. Biol 311, 487-499.

Kakihara, K., Shinmyozu, K., Kato, K., Wada, H. \& Hayashi, S. (2008). Conversion of plasma membrane topology during epithelial tube connection requires Arf-like 3 GTPase in Drosophila. Mechanisms of Development 125, 325-336.

Karagiosis, S. \& Ready, D. (2004). Moesin contributes an essential structural role in Drosophila photoreceptor morphogenesis. Development 131, 725-32.

Keleman, K. \& Dickson, B. (2001). Short- and Long-Range Repulsion by the Drosophila Unc5 Nectrin Receptor. Neuron 32, 605-617.

Kerman, B., Chesire, A., Myat, M. \& Andrew, D. (2008). Ribbon modulates apical membrane during tube elongation through Crumbs and Moesin. Developmental Biology 320, 278-288.

Kidd, T., Bland, K. \& Goodman, C. (1999). Slit is the midline repellent for the robo receptor in Drosophila. Cell 96, 785-94.

Kivirikko, K. \& Pihlajaniemi, T. (1998 ). Collagen hydroxylases and the protein disulfide isomerase subunit of prolyl 4-hydroxylases. Adv Enzymol Relat Areas Mol Biol 72, 325-98.

Knox, J., Moyer, K., Yacoub, N., Soldaat, C., Komosa, M., Vassilieva, K., Wilk, R., Hu, J., de Lourdes Vasquez Pas, L., Syed, Q. et al. (2011). Syndecan contributes to heart cell specification and lumen formation during Drosophila cardiogenesis. Developmental Biology 356, 279-290.

Koh, W., Mahan, R. \& Davis, G. (2008). Cdc42- and Rac1-mediated endothelial lumen formation requires Pak2, Pak4 and Par3, and PKC-dependent signaling. J Cell Sci 121, 989-1001.

Koh, W., Sachidanandam, K., Stratman, A., Sacharidou, A., Mayo, A., Murphy, E., Cheresh, D. \&Davis, G. (2009). Formation of endothelial lumens requires a coordinated $\mathrm{PKC}_{\mathrm{E}-}$, Src-, Pak- and Raf- kinase-dependent signaling cascade downstream of Cdc42 activation. J Cell Sci 122, 1812-1822. 
Kracklauer, M., Banks, S., Xie, X., Wu, Y. \& Fischer, J. (2007). Drosophila klaroid encodes a SUN domain protein required for Klarsicht localization to the nuclear envelope and nuclear migration in the eye. Fly (Austin) 1, 75-85.

Laprise, P., Paul, S., Boulanger, J., Robbins, R., Beitel, G. J. \& Tepass, U. (2010). Epithelial polarity proteins regulate Drosophila tracheal tube size in parallel to the luminal matrix pathway. Current Biology 20, 55-61.

Lee, M., Lee, S., Zadesh, A. D. \& Kolodziej, P. A. (2003). Distinct sites in E-cadherin regulate different steps in Drosophila tracheal tube fusion. Development 130, 5989-5999.

Lee, S. \& Kolodziej, P. A. (2002). The plakin Short Stop and the RhoA GTPase are requiered for E- cadherin-dependent apical surface remodeling during tracheal tube fusion. Development 129, 1509-1520.

Levi, B., Ghabrial, A. \& Krasnow, M. A. (2006). Drosophila talin and integrin genes are required for maintenance of tracheal terminal branches and luminal organization. Development 133, 2383-93.

Liu, J., Fraser, S., Faloon, P., Rollins, E., Vom Berg, J., Starovic-Subota, O., Laliberte, A., Chen, J., Serluca, F. \& Childs, S. (2007). A betaPix pak2a signaling pathway regulates cerebral vascular stability in zebrafish. Proc Natl Acad Sci USA 104, 13990-13995.

Llimargas, M., Strigini, M., Katidou, M., Karagogeos, D. \& Casanova, J. (2004). Lachesin is a component of a septate junction-based mechanism that controls tube size and epithelial integrity in the Drosophila tracheal system. Development 131, 181-190.

Lubarsky, B. \& Krasnow, M. A. (2003). Tube morphogenesis: making and shaping biological tubes. Cell 112, 19-28.

Luschnig, S., Batz, T., Armbruster, K. \& Krasnow, M. A. (2006). Serpentine and Vermiform encode matrix proteins with chitin binding and deacetylation domains that limit tracheal tube length in Drosophila. Current Biology 16, 186-194.

Malbon, C. (2005). G proteins in development Nat Rev Mol Cell Biol 6, 689-701.

Maruyama, R., Grevengoed, E., Stempniewicz, P. \& Andrew, D. (2011). Genome-wide analysis reveals a major role in cell fate maintenance and an unexpected role in endoreduplication for the Drosophila FoxA gene Fork head. PLoS One 6, e20901.

Massarwa, R., Schejter, E. \& Shilo, B. (2009). Apical secretion in epithelial tubes of the Drosophila embryo is directed by the Formin-family protein Diaphanous. Dev Cell. $16,877-888$.

McClatchey, A. \& Fehon, R. (2009). Merlin and the ERM proteins--regulators of receptor distribution and signaling at the cell cortex. Trends Cell Biol 19, 198-206.

Medioni, C., Astier, M., Zmojdzian, M., Jagla, K. \& Semeriva, M. (2008). Genetic control of cell morphogenesis during Drosophila melanogaster cardiac tube formation. J Cell Biol 182, 249-261.

Mosley-Bishop, K. L., Li, Q., Patterson, L. \& Fischer, J. A. (1999). Molecular analysis of the klarsicht gene and its role in nuclear migration within differentiating cells of the Drosophila eye. Current Biology 9, 1211-1220.

Moussian, B., Tang, E., Tonning, A., Helms, S., Schwarz, H., Nusslein-Volhard, C. \& Uv, A. (2006). Drosophila Knickkopf and Retroactive are needed for epithelial tube growth and cuticle differentiation through their specific requirement for chitin filament organization. Development 133, 163-171.

Myat, M. M. \& Andrew, D. J. (2000a). Organ shape in the Drosophila salivary gland is controlled by regulated, sequential internalization of the primordia. Development 127, 679-691.

Myat, M. M. \& Andrew, D. J. (2000b). Fork head prevents apoptosis and promotes cell shape change during formation of the Drosophila salivary glands. Development 127, 4217-4226. 
Myat, M. M. \& Andrew, D. J. (2002). Epithelial tube morphology is determined by the polarized growth and delivery of apical membrane. Cell 111, 879-891.

Nelson, K., Furuse, M. \& Beitel, G. (2010). The Drosophila Claudin Kune-kune is required for septate junction organization and tracheal tube size control. Genetics 185, 831-839.

Ohshiro, T., Emori, Y. \& Saigo, K. (2002). Ligand-dependent activation of breathless FGF receptor gene in Drosophila developing trachea. . Mechanisms of Development 114, 3-11.

Ostrowski, S., Dierick, H. \& Bejsovec, A. (2002). Genetic control of cuticle formation during embryonic development of Drosophila melanogaster. Genetics 161, 171-182.

Paul, S. M., Ternet, M., Salvaterra, P. M. \& Beitel, G. J. (2003). The Na+/K+ ATPase is required for septate junction function and epithelial tube-size control in the Drosophila. Development 130, 4963-4974.

Pellikka, M., Tanentzapf, G., Pinto, M., Smith, C., McGlade, C. J., Ready, D. F. \& Tepass, U. (2002). Crumbs, the Drosophila homologue of human CRB1/RP12, is essential for photoreceptor morphogenesis. Nature 416, 143-149.

Pirraglia, C. \& Myat, M. M. (2010). Genetic Regulation of Salivary Gland Development in Drosophila melanogaster. In Salivary Glands: Development, Adaptations and Disease., vol. 14 (ed. A. Tucker and I. Miletich), pp. 32-47: S. Karger.

Pirraglia, C., Walters, J. \& Myat, M. M. (2010). Pak1 control of E-cadherin endocytosis regulates salivary gland lumen size and shape. Development 137, 4177-4189.

Qian, L., Liu, J. \& Bodmer, R. (2005). Slit and Robo Control Cardiac Cell Polarity and Morphogenesis. Current Biology 15, 2271-2278.

Reim, I. \& Frasch, M. (2010). Genetic and genomic dissection of cardiogenesis in the Drosophila model. Pediatr Cardiol 31, 325-334.

Rothberg, J., Jacobs, J., Goodman, C. \& Artavanis-Tsakonas, S. (1990). Slit: an extracellular protein necessary for development of the midline glia and commissural axon pathway contains both EGF and LRR domains. Genes and Development 4, 2169-2187.

Santiago-Martinez, E., Soplop, N., Patel, R. \& Kramer, S. (2008). Repulsion by Slit and Roundabout prevents Shotgun/E-cadherin-mediated cell adhesion during Drosophila heart tube lumen formation. J Cell Biol 182, 241-248.

Satoh, A., O'Tousa, J., Ozaki, K. \& Ready, D. (2005). Rab11 mediates post-Golgi trafficking of rhodopsin to the photosensitive apical membrane of Drosophila photoreceptors. Development 132, 1487-97.

Seshaiah, P., Miller, B., Myat, M. M. \& Andrew, D. J. (2001). Pasilla, the Drosophila homologue of the human Nova-1 and Nova-2 proteins, is required for normal secretion in the salivary gland. Developmental Biology 239, 309-22.

Shim, K., Blake, K. J., Jack, J. \& Krasnow, M. A. (2001). The Drosophila ribbon gene encodes a nuclear BTB domain protein that promotes epithelial migration and morphogenesis. Development 128, 4923-4933.

Strillic, B., Kucera, T., Eglinger, J., Huges, M., McNagny, K., Tsukita, S., Dejana, E., Ferrara, N. \& Lamert, E. (2009). The molecular basis of vascular lumen formation in the developing mouse aorta. Developmental Cell 17, 505-515.

Sutherland, D., Samakovlis, C. \& Kransnow, M. A. (1996). Branchless encodes a Drosophila FGF homolog that controls tracheal cell migration and the pattern of branching. Cell 87, 1091-1101.

Swanson, L., Yu, M., Nelson, K., Laprise, P., Tepass, U. \& Beitel, G. (2009). Drosophila convoluted/dALS is an essential gene required for tracheal tube morphogenesis and apical matrix organization. Genetics 181, 1281-1290.

Tao, Y. \& Schulz, R. (2007). Heart development in Drosophila. Seminars in Cell $\mathcal{E}$ Developmental Biology 18, 3-15. 
Tepass, U., Gruszynski-DeFeo, E., Haag, T. A., Omatyar, L., Torok, T. \& Hartenstein, V. (1996). Shotgun encodes Drosophila E-cadherin and is preferentially required during cell rearrangement in the neurectoderm and other morphogenetically active epithelia. Genes \& Development 10, 672-685.

Tepass, U. \& Knust, E. (1990). Phenotypic and developmental analysis of mutations at the crumbs locus, a gene required for the development of epithelia in Drosophila melanogaster. Roux's Arch Dev Biol 199, 189-206.

Tepass, U., Tanentzapf, G., Ward, R. \& Fehon, R. (2001). Epithelial cell polarity and cell junctions in Drosophila. Annu Rev Genet 35, 747-784.

Tonning, A., Hemphala, J., Tang, E., Nannmark, U., Samakovlis, C. \& Uv, A. (2005). A transcient luminal chitinous matrix is required to model epithelial tube diameter in the Drosophila Trachea. Developmental Cell 9, 423-430.

Tsarouhas, V., Senti, K., Jayaram, S., Tiklova, K., Hemphala, J., Adler, J. \& Samakovlis, C. (2007). Sequential pulses of apical epithelial secretion and endocytosis drive airway maturation in drosophila. Dev Cell 13.,214-225.

von Hilchen, C., Hein, I., Technau, G. \& Altenhein, B. (2010). Netrins guide migration of distinct glial cells in the Drosophila embryo. Development 137, 1251-1262.

Wang, S., Jayaram, S., Hemphala, J., Senti, K., Tsarouhas, V., Jin, H. \& Samakovlis, C. (2006). Septate- junction-dependent luminal deposition of chitin deacetylases restricts tube elongation in the Drosophila trachea. Current Biology 16, 180-185.

Wang, S., Tsahouras, V., Xylourgidis, N., Sabri, N., Tiklova, K., Nautiyal, N., Gallio, M. \& Samakovlis, C. (2009). The tyrosine kinase Stitcher activates Grainy head and epidermal wound healing in Drosophila. Nat Cell Biol 11, 890-895.

Welte, M. A., Gross, S. P., Postner, M., Block, S. M. \& Wieschaus, E. F. (1998). Developmental Regulation of Vesicle Transport in Drosophila Embryos: Forces and Kinetics. Cell 92, 547-557.

Wodarz, A., Hinz, U., Engelbert, M. \& Knust, E. (1995). Expression of Crumbs confers apical character on plasma membrane domains of ectodermal epithelia of Drosophila. Cell 82, 67-76.

Wu, V. M., Schulte, J., Hirschi, A., Tepass, U. \& Beitel, G. J. (2004). Sinuous is a Drosophila claudin required for septate junction organization and epithelial tube size control. The Journal of Cell Biology 164, 313-323.

Yi, P., Han, Z., Li, X. \& Olson, E. (2006). The Mevalonate Pathway Controls Heart Formation in Drosophila by Isoprenylation of G $\gamma 1$. Science 313, 1301-1303.

Yi, P., Johnson, A., Han, Z., Wu, J. \& Olson, E. (2008). Heterotrimeric G Proteins Regulate a Noncanonical Function of Septate Junction Proteins to Maintain Cardiac Integrity in Drosophila. Developmental Cell 15, 704-713.

Zhan, Y., Maung, S. W., Shao, B. \& Myat, M. M. (2010). The bHLH transcription factor, hairy, refines the terminal cell fate in the Drosophila embryonic trachea. PLoS One 5, e14134.

Zhang, J., Felder, A., Liu, Y., Guo, L., Lange, S., Dalton, N., Gu, Y., Peterson, K., Mizisin, A., Shelton, G. et al. (2010). Nesprin 1 is critical for nuclear positioning and anchorage. Hum Mol Genet 19, 329-41.

Zhang, Q., Skepper, J., Yang, F., Davies, J., Hegyi, L., Roberts, R., Weissberg, P., Ellis, J. \& Shanahan, C. (2001). Nesprins: a novel family of spectrin-repeat-containing proteins that localize to the nuclear membrane in multiple tissues. J Cell Sci 114, 4485-98. 


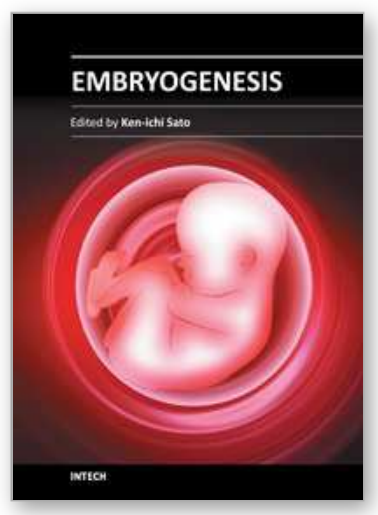

\author{
Embryogenesis \\ Edited by Dr. Ken-Ichi Sato
}

ISBN 978-953-51-0466-7

Hard cover, 652 pages

Publisher InTech

Published online 20, April, 2012

Published in print edition April, 2012

The book "Embryogenesis" is a compilation of cutting edge views of current trends in modern developmental biology, focusing on gametogenesis, fertilization, early and/or late embryogenesis in animals, plants, and some other small organisms. Each of 27 chapters contributed from the authorships of world-wide 20 countries provides an introduction as well as an in-depth review to classical as well as contemporary problems that challenge to understand how living organisms are born, grow, and reproduce at the levels from molecule and cell to individual.

\title{
How to reference
}

In order to correctly reference this scholarly work, feel free to copy and paste the following:

$\mathrm{Na}$ Xu, Carolyn Pirraglia, Unisha Patel and Monn Monn Myat (2012). Mechanisms of Lumen Development in Drosophila Tubular Organs, Embryogenesis, Dr. Ken-Ichi Sato (Ed.), ISBN: 978-953-51-0466-7, InTech, Available from: http://www.intechopen.com/books/embryogenesis/mechanisms-of-lumen-development-indrosophila-tubular-organs

\section{INTECH}

open science | open minds

\section{InTech Europe}

University Campus STeP Ri

Slavka Krautzeka 83/A

51000 Rijeka, Croatia

Phone: +385 (51) 770447

Fax: +385 (51) 686166

www.intechopen.com

\section{InTech China}

Unit 405, Office Block, Hotel Equatorial Shanghai

No.65, Yan An Road (West), Shanghai, 200040, China

中国上海市延安西路65号上海国际贵都大饭店办公楼 405 单元

Phone: +86-21-62489820

Fax: +86-21-62489821 
(C) 2012 The Author(s). Licensee IntechOpen. This is an open access article distributed under the terms of the Creative Commons Attribution 3.0 License, which permits unrestricted use, distribution, and reproduction in any medium, provided the original work is properly cited. 